\title{
Assessing the Impact of the COVID-19 Pandemic on Postpartum Contraception Uptake
}

PAIGE RATTNER

CHELSEA DEBOLT

LORRAINE TONER

GUILLAUME STOFFELS

SHARON GERBER

LUCIANA VIEIRA

*Author affiliations can be found in the back matter of this article

\section{ABSTRACT}

Background: Due to COVID-19, telemedicine was incorporated into postpartum care to reduce transmission risk.

Research Question: Our objective was to compare postpartum contraception uptake between March 1, 2019-May 31, 2019 and March 1, 2020-May 31, 2020.

Study Design/Methods: This is a retrospective cohort study of postpartum patients who delivered at the Mount Sinai Hospital System between March 1, 2019-May 31, 2019 and March 1, 2020-May 31, 2020. We recorded postpartum contraception uptake at hospital discharge and at outpatient postpartum visits. We compared four categories: short-acting reversible contraception (SARC), long-acting reversible contraception (LARC), "barrier methods/other" (condoms, diaphragms, spermicides), and sterilization. The 2019 and 2020 patient cohorts did not differ significantly in terms of maternal age, gravidity, ethnicity, or insurance type. This study was approved by the Mount Sinai IRB.

Results: 1,106 patients were included in our analysis, 443 and 663 who delivered in 2019 and 2020 respectively. There were no significant differences in the number of patients offered and accepting contraception at hospital discharge and postpartum visit between the 2019 and 2020 groups. Type of contraception given at postpartum visit differed significantly between 2019 and 2020 ( $p=0.006$ ), with the 2020 group more likely to use SARC (55 vs. $42 \%$ ) and less likely to use LARC (15 vs. 24\%). Notably, $26 \%$ of the 2020 group had telehealth postpartum visits (vs. $0 \%$ in 2019). Within the 2020 group, type of contraception differed significantly between in-person and telehealth visits $(p=0.001)$. Patients who had telehealth visits were less likely to use "barrier/other" methods (14 vs. 32\%) and more likely to use SARC (67 vs. 51\%).

Conclusions: Despite COVID-19, overall uptake of postpartum contraception did not change significantly; however, the type of contraception differed significantly, particularly at telehealth visits. Additional research is needed to understand why telehealth postpartum visits may increase the uptake of short acting contraception and decrease barrier methods.
CORRESPONDING AUTHOR:

\section{Paige Rattner}

Icahn School of Medicine at Mount Sinai, US

paige.rattner@icahn.mssm.edu

KEYWORDS:

COVID-19, contraception, postpartum contraception, telemedicine

TO CITE THIS ARTICLE: Rattner P, DeBolt C, Toner L, Stoffels G, Gerber S, Vieira L. Assessing the Impact of the COVID-19 Pandemic on Postpartum Contraception Uptake. ISMMS Journal of Science and Medicine. 2021; 1(2): 3, pp. 1-2. DOI: https:// doi.org/10.29024/ijsm.50 
The authors have no competing interests to declare.

\section{AUTHOR AFFILIATIONS}

\section{Paige Rattner}

Icahn School of Medicine at Mount Sinai, US

\section{Chelsea DeBolt}

Icahn School of Medicine at Mount Sinai, US

\section{Lorraine Toner}

Icahn School of Medicine at Mount Sinai, US

\section{Guillaume Stoffels}

Icahn School of Medicine at Mount Sinai, US

Sharon Gerber

Icahn School of Medicine at Mount Sinai, US

\section{Luciana Vieira}

Icahn School of Medicine at Mount Sinai, US

TO CITE THIS ARTICLE:

Rattner P, DeBolt C, Toner L, Stoffels G, Gerber S, Vieira L. Assessing the Impact of the COVID-19 Pandemic on Postpartum Contraception Uptake. ISMMS Journal of Science and Medicine. 2021; 1(2): 3, pp. 1-2. DOI: https:// doi.org/10.29024/ijsm.50

(c) 2021 The Author(s). This is an open-access article distributed under the terms of the Creative Commons Attribution 4.0 International License (CC-BY 4.0), which permits unrestricted use, distribution, and reproduction in any medium, provided the original author and source are credited. See http://creativecommons.org/ licenses/by/4.0\%.

ISMMS Journal of Science and 